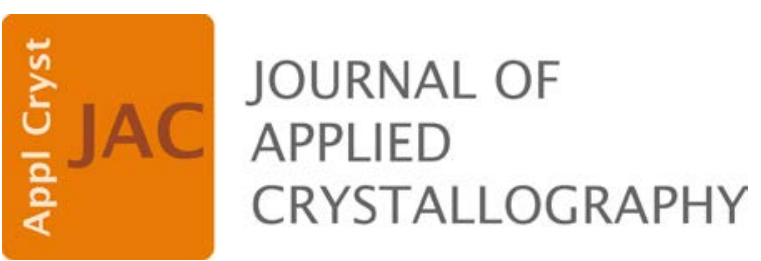

Volume 49 (2016)

Supporting information for article:

Quantitative X-ray pair distribution function analysis of nanocrystalline calcium silicate hydrates: a contribution to the understanding of cement chemistry

Sylvain Grangeon, Alejandro Fernandez-Martinez, Alain Baronnet, Nicolas Marty, Agnieszka Poulain, Erik Elkaïm, Cédric Roosz, Stéphane Gaboreau, Pierre Henocq and Francis Claret 
Table S1 All parameters refined during PDF data simulation but those reported in the main text. The corresponding input file is given in Figure S1.

\begin{tabular}{|c|c|c|c|c|c|c|c|}
\hline \multirow[t]{2}{*}{$\mathrm{Ca} / \mathrm{Si}$ ratio } & \multirow[t]{2}{*}{ factor } & \multirow[t]{2}{*}{$\left(\AA^{2}\right)$} & \multirow[t]{2}{*}{$(\AA)$} & & $\mathrm{Ca}$ and $\mathrm{Si}$ atoms & $\mathrm{O}$ atoms & \\
\hline & & & & $\mathrm{u}_{11}$ and $\mathrm{u}_{22}\left(\AA^{2}\right)$ & $\mathrm{u}_{33}\left(\AA^{2}\right)$ & $\mathrm{u}_{11}$ and $\mathrm{u}_{22}\left(\AA^{2}\right)$ & $\mathrm{u}_{33}\left(\AA^{2}\right)$ \\
\hline 0.6 & 1.08 & 2.52 & 28 & 0.010 & 0.002 & 0.004 & 0.04 \\
\hline 0.8 & 1.17 & 2.54 & 37 & 0.006 & 0.008 & 0.007 & 0.07 \\
\hline 1 & 1.17 & 2.52 & 41 & 0.006 & 0.009 & 0.007 & 0.06 \\
\hline 1.2 & 1.18 & 2.52 & 30 & 0.005 & 0.008 & 0.008 & 0.06 \\
\hline
\end{tabular}

Notes: delta2 is a quadratic atomic correlation factor. $\mathrm{U}_{33}$ associated to layer oxygen atoms are higher than the corresponding $U_{11}$ and $U_{22}$ values, certainly because the $\mathbf{c}$ lattice parameter was fixed during the modelling procedure so as to reduce the number of free parameters. 
Table S2 Detail of the four PDF simulations performed on C-S-H samples having a target $\mathrm{Ca} / \mathrm{Si}$ of 1.2.

\begin{tabular}{lllll}
\hline Parameter & \multicolumn{4}{c}{ Constraints on occupancy of Si sites } \\
\cline { 2 - 5 } & $\begin{array}{l}\text { Bridging } \\
\text { sites refined }\end{array}$ & $\begin{array}{l}\text { Paired sites } \\
\text { refined }\end{array}$ & $\begin{array}{l}\text { Equal occupancy of } \\
\text { bridging and paired } \\
\text { sites }\end{array}$ & $\begin{array}{l}\text { Bridging and paired } \\
\text { sites refined } \\
\text { independently }\end{array}$ \\
\hline$a(\AA)$ & $6.685(8)$ & $6.678(8)$ & $6.680(8)$ & $6.683(8)$ \\
Scale factor & $1.16(6)$ & $1.25(7)$ & $1.23(7)$ & $1.18(7)$ \\
Delta2 $\left(\AA^{2}\right)$ & $2.5(1)$ & $2.5(1)$ & $2.5(1)$ & $2.5(1)$ \\
Spdiameter $(\AA)$ & $29(4)$ & $31(4)$ & $31(4)$ & $30(4)$ \\
$z$-coordinate of bridging $\mathrm{Si}^{\S}$ & $0.065(4)$ & $0.067(1)$ & $0.0678(2)$ & $0.066(4)$ \\
Ca and Si $\mathrm{U}_{11}$ and $\mathrm{U}_{22}\left(\AA^{2}\right)$ & $0.005(1)$ & $0.006(1)$ & $0.005(1)$ & $0.005(1)$ \\
Ca and Si $\mathrm{U}_{33}\left(\AA^{2}\right)$ & $0.007(3)$ & $0.011(4)$ & $0.011(4)$ & $0.008(3)$ \\
O $\mathrm{U}_{11}$ and $\mathrm{U}_{22}\left(\AA^{2}\right)$ & $0.007(5)$ & $0.007(2)$ & $0.008(2)$ & $0.008(2)$ \\
O U $\mathrm{U}_{33}\left(\AA^{2}\right)$ & $0.06(1)$ & $0.06(1)$ & $0.06(1)$ & $0.06(1)$ \\
Occupancy of bridging Si & $0.37(9)$ & $1^{*}$ & $0.78(4)^{\S}$ & $0.45(11)$ \\
Occupancy of paired $\mathrm{Si}$ & $1^{*}$ & $0.75(5)$ & $0.78(4)^{\S}$ & $0.91(6)$ \\
$\mathrm{R}_{\mathrm{WP}}$ & $32.70 \%$ & $34.66 \%$ & $35.74 \%$ & $32.41 \%$ \\
\hline
\end{tabular}

Notes: ${ }^{\S}$ Relative to the model given in Figure S1; ${ }^{*}$ fixed; ${ }^{\$}$ covaried 
Figure S1: Typical PdfGUI input file used to model PDF patterns. Greyed parameters are those which were possibly refined (see main text and Table SI1).

\section{Phase Configuration}

\begin{tabular}{|c|c|c|c|c|c|c|c|c|c|c|c|}
\hline & & & & b & 73 & & c 22.487 & & & & \\
\hline slpt & tho 90 . & & & beta & 90.0 & gamm & $\mathrm{ms} 123.25$ & & & & \\
\hline & ile $\mathrm{fo}$. & 097 & 0 & & & & & & & & \\
\hline & delta & 0.0 & & & delta2 257 & & spdismete & er & & & \\
\hline & srati & io 10 & & & reut 0.0 & & stepcu & Os & & & \\
\hline & luded P & Pairs a & Il-all & & & & & & & & \\
\hline & elem & $x$ & y & $z$ & u11 & u22 & 433 & u12 & u13 & 423 & occ \\
\hline 1 & Si & 0.9087 & 0.7531 & 0.0712 & 0,0026164 & 0.9026164 & 0,00799335 & 0.0 & 0.0 & 0.0 & 10 \\
\hline 2 & Si & 0.9087 & 0.7531 & 09238 & 0.0026164 & 0.0026164 & 0.00799335 & 0.0 & 0.0 & 0.0 & 10 \\
\hline 3 & Si & 0.4087 & 0.7531 & 0.5712 & 0.0026164 & 0,0026164 & 0.00799335 & 0.0 & 0.0 & 0.0 & 10 \\
\hline 4 & Si & 0.4087 & 0.7531 & 0.4238 & 10.0026164 & 0.0026164 & 0.00799335 & 0.0 & 0.0 & 0.0 & 10 \\
\hline 5 & 0 & 0.771 & 0.5059 & 0.0942 & 0.00461025 & 0.00461025 & 0.0420663 & 0.0 & 0.0 & 0.0 & 1.0 \\
\hline 6 & 0 & 0.771 & 0.5059 & 0.9058 & 0.00461025 & 0,00461025 & 0.0429663 & 0.0 & 0.0 & 0.0 & 10 \\
\hline 7 & 0 & 0.271 & 0.5059 & 0.5942 & 0.00461025 & 0,00461025 & 0.0429663 & 0.0 & 0.0 & 0.0 & 10 \\
\hline 8 & 0 & 0.271 & 0.5059 & 0.4058 & 0.00461025 & 0.00461025 & 0.0429663 & 0.0 & 0.0 & 0.0 & 1.0 \\
\hline 9 & 0 & 0.759 & 0.178 & 0.1327 & 0.00461025 & 0,00461025 & 0.0429663 & 0.0 & 0.0 & 0.0 & 1.0 \\
\hline 10 & 0 & 0.759 & 0.178 & 0.8673 & 0.00461025 & 0,00461025 & 0.0420663 & 0.0 & 0.0 & 0.0 & 10 \\
\hline 11 & 0 & 0.259 & 0.178 & 0.6327 & 0.00461025 & 0.00461025 & 0.0429663 & 0.0 & 0.0 & 0.0 & 10 \\
\hline 12 & 0 & 0.259 & 0.178 & 0.3673 & 0.00461025 & 0,00461025 & 0.0429663 & 0.0 & 0.0 & 0.0 & 1.0 \\
\hline 13 & 0 & 0.985 & 0.5369 & 0.1982 & 0.00461025 & 0.00461025 & 0.0429663 & 0.0 & 0.0 & 0.0 & 10 \\
\hline 14 & 0 & 0985 & 0.5369 & 0.8018 & 0.00461025 & 0.00461025 & 0.0429663 & 0.0 & 0.0 & 0.0 & 1.0 \\
\hline 15 & 0 & 0.485 & 0.5369 & 0.6982 & 0.00461025 & 0.00461025 & 0,9429663 & 0.0 & 0.0 & 0.0 & 1.0 \\
\hline 16 & 0 & 0.485 & 0.5369 & 0.3018 & 0.00461025 & 0.00461025 & 0.0429663 & 0.0 & 0.0 & 0.0 & 1.0 \\
\hline 17 & 0 & 0.519 & 0.3063 & 0.1942 & 0.00461025 & 0.00461025 & 0.0420663 & 0.0 & 0.0 & 0.0 & 1.0 \\
\hline 18 & 0 & 0.519 & 0.3063 & 0.8058 & 0.00461025 & D.00461025 & 0.0429663 & 0.0 & 0.0 & 0.0 & 10 \\
\hline 19 & 0 & 0.019 & 0.3063 & 0.6942 & 0.00461025 & 0.00461025 & 0.0420663 & 0.0 & 0.0 & 0.0 & 1.0 \\
\hline 20 & 0 & 0.019 & 0.3063 & 0.3058 & 0.00451025 & 0,00461025 & 0.0429663 & 0.0 & 0.0 & 0.0 & 1.0 \\
\hline 21 & 0 & 0.894 & 0.746 & 0.0 & 0,00461025 & 0.00461025 & 0.0429663 & 0.0 & 0.0 & 0.0 & 10 \\
\hline 22 & 0 & 0.394 & 0.746 & 0.5 & 0.00461025 & 0.00461025 & 0.0420663 & 0.0 & 0.0 & 000 & 10 \\
\hline 23 & 0 & 0.188 & 0.893 & 0.094 & 0.00861025 & 0.00461025 & 0.0429663 & 100 & 0.0 & 0.0 & 1.0 \\
\hline 24 & 0 & 0.188 & 0.893 & 0.906 & 0.00461025 & 0.00461025 & 0.0429663 & 000 & 0.0 & 0.0 & 1.0 \\
\hline 25 & 0 & 0.688 & 0.893 & 0.594 & 0.00461025 & 0.00461025 & 0.0420663 & 000 & 0.0 & 0.0 & 1.0 \\
\hline 26 & 0 & 0.688 & 0.893 & 0.406 & 0.00461025 & 0.00461025 & 0.0429663 & 000 & 0.0 & 0.0 & 1.0 \\
\hline 27 & 0 & 0.27 & 0.434 & 0.0939 & 0.00461025 & 0.00461025 & 0.0420663 & 100 & 0.0 & 0.0 & 1.0 \\
\hline 28 & 0 & 0.27 & 0.434 & 0.9061 & 000461025 & 0.00461025 & 0.0420663 & 100 & 0.0 & 0.0 & 1.0 \\
\hline 29 & 0 & 0.77 & 0.434 & 0.5939 & 0.00461025 & 0.00461025 & 0.0429663 & 0.0 & 0.0 & 0.0 & 1.0 \\
\hline 30 & 0 & 0.77 & 0.434 & 0.4061 & 0.00461025 & 0.00461025 & 0.0429663 & 1000 & 0.0 & 0.0 & 1.0 \\
\hline 31 & 0 & 0.77 & 0.86 & 0.0951 & 0.00461025 & 0.00461025 & 0.0420663 & 0.0 & 0.0 & 0.0 & 1.0 \\
\hline 32 & 0 & 0.77 & 0.86 & 0.9049 & 0.00461025 & 0.00461025 & 0.0429663 & 0.0 & 0.0 & 0.0 & 1.0 \\
\hline 33 & 0 & 0.27 & 0.86 & 0.5951 & 0.00461025 & 0.00461025 & 0.0429663 & 0.0 & 0.0 & 0.0 & 1.0 \\
\hline 34 & 0 & 0.27 & 0.86 & 0.4049 & 0.00461025 & 0.00461025 & 0.0420653 & 1000 & 0.0 & 0.0 & 1.0 \\
\hline 35 & 0 & 0.523 & 0.811 & 0.1951 & 0.0461025 & 0.00461025 & 0.0429663 & 0.0 & 0.0 & 0.0 & 1.0 \\
\hline 36 & 0 & 0.523 & 0.811 & 0.8049 & $0.00 \pm 51025$ & 0.00461025 & 0.0420663 & 0.0 & 0.0 & 0.0 & 1.0 \\
\hline 37 & 0 & 0.023 & 0.811 & 0.6951 & 0.00461025 & 0.00461025 & 0.0420663 & 10.0 & 0.0 & 0.0 & 1.0 \\
\hline 38 & 0 & 0.023 & 0.811 & 0.3049 & 0.00461025 & 0.00461025 & 0.0429663 & 0.0 & 0.0 & 0.0 & 1.0 \\
\hline 39 & 0 & 0.987 & 0.0459 & 0.1985 & 0.00461025 & 0.00461025 & 0.0429663 & 0.0 & 0.0 & 0.0 & 1.0 \\
\hline 40 & 0 & 0.987 & 0.0459 & 0.8015 & 0.00461025 & 0.00461025 & 0.0420663 & 1000 & 0.0 & 0.0 & 1.0 \\
\hline 41 & 0 & 0.487 & 0.0459 & 0.6985 & 0.00461025 & 0.00461025 & 0.0429663 & 100 & 0.0 & 0.0 & 1.0 \\
\hline 42 & 0 & 0.487 & 0.0459 & 0.3015 & 0.00461025 & 0.00461025 & 0.0420663 & 0.0 & 0.0 & 0.0 & 1.0 \\
\hline 43 & Si & 0.7581 & 0.3862 & 0.1574 & 0.0026164 & 0.0026164 & 0.00799335 & 0.0 & 0.0 & 0.0 & 1.0 \\
\hline 44 & Si & 0.7581 & 0.3862 & 0.8426 & 0.0026164 & 0.0026164 & 0,00799335 & 0.0 & 0.0 & 0.0 & 10 \\
\hline 45 & Si & 0.2581 & 0.3862 & 0.6574 & 0.0026164 & 00026164 & 0.00799335 & 10.0 & 0.0 & 0.0 & 10 \\
\hline 46 & si & 0.2581 & 0.3862 & 0,3426 & 0.0026164 & 0.0026164 & 0.00799335 & 0.0 & 0.0 & 0.0 & 10 \\
\hline 47 & Si & 0.7592 & 0.9697 & 0.1577 & 0.0026164 & 0.0026164 & 0.00799335 & 1000 & 0.0 & 0.0 & 1.0 \\
\hline 48 & Si & 0.7592 & 0.9697 & 0.8423 & 0.0026164 & 0.0026164 & 0.00799335 & 0.0 & 0.0 & 0.0 & 1.0 \\
\hline 49 & si & 0.2592 & 0.9697 & 0.6577 & 0,0026164 & 0.0026164 & 0,00799335 & 0.0 & 0.0 & 0.0 & 1.0 \\
\hline 50 & si & 0.2592 & 0.9697 & 0.3423 & 0.0026164 & 0.0026164 & 0.00799335 & 0.0 & 0.0 & 0.0 & 1.0 \\
\hline 51 & Co & 0.2651 & 0.4328 & 0.2056 & 0.0026164 & 0.0026164 & 0.00799335 & 10.0 & 000 & 0.0 & 1.0 \\
\hline 52 & Ca & 0.2651 & 0.4328 & 0.7944 & 0.0026164 & 0,0026164 & 0,00799335 & 10.0 & 0.0 & 0.0 & 1.0 \\
\hline 53 & $\mathrm{Ca}$ & 0.7651 & 0.4323 & 0.7056 & 0.0026164 & 0.0026164 & 0,00799335 & 0.0 & 000 & 0.0 & 1.0 \\
\hline 54 & Co & 0.7651 & 0.4328 & 0.2944 & 0.0026164 & 0.0026164 & 0.00799335 & 0.0 & 0.0 & 0.0 & 1.0 \\
\hline 55 & Ca & 0.7499 & 0.9228 & 0.2935 & 0.9026164 & 0.0026164 & 0.00799335 & 1000 & 0.0 & 0.0 & 1.0 \\
\hline 56 & ca & 0.7499 & 09228 & 0.7065 & 0.0026164 & 0.0026164 & 0,00799335 & 0.0 & 0.0 & 0.0 & 1.0 \\
\hline 57 & Ca & 0.2499 & 0.9228 & 0.7935 & 0.0026164 & 0.0026164 & 0.00799335 & 0.0 & 0.0 & 0.0 & 1.0 \\
\hline 58 & Ca & 0.2499 & 0.9228 & 0.2065 & 0.0026164 & 0,0026164 & 0.00799335 & 0.0 & 0.0 & 0.0 & 1.0 \\
\hline 59 & 0 & 0.427 & 0.219 & 0.0 & 0.0524101 & 0.0524101 & 0.228507 & 0.0 & 0.0 & 0.0 & 0.25 \\
\hline 60 & 0 & 0927 & 0.219 & 0.5 & 0.0524101 & 0.0524101 & 0.228507 & 0.0 & 0.0 & 0.0 & 0.25 \\
\hline 61 & 0 & 0.879 & 0.237 & 0.0 & 0.0524101 & 0.0524101 & 0.228507 & 10.0 & 0.0 & 0.0 & 0.25 \\
\hline 62 & 0 & 0.379 & 0.237 & 0.5 & 0.0524101 & 0.0524101 & 0.228507 & 0.0 & 0.0 & 0.0 & 0.25 \\
\hline 63 & 0 & 0.422 & 0.8 & 0.0 & 0.0524101 & 0.0524101 & 0.228507 & 0.0 & 0.0 & 0.0 & 0.25 \\
\hline 64 & 0 & 0.922 & 0.8 & 0.5 & 0.0524101 & 0.0524101 & 0.228507 & 0.0 & 0.0 & 0.0 & 0.25 \\
\hline
\end{tabular}

